\author{
Francesco Zanchini di Castiglionchio \\ (già professore ordinario di Diritto canonico nell'Università degli Studi \\ di Teramo, Facoltà di Giurisprudenza)
}

\title{
"A chiare lettere - Confronti" • A proposito di guerra o pace ai tribunali ecclesiastici esistenti in Italia. Quale cultura di riforme organiche sta dietro l'attuale pontificato?
}

Regarding peace of war to the italian ecclesiastical courts.

Which is the methodical reform background of pope Francis?

\begin{abstract}
The accentuated tendency of the part of the Vatican (MP Mitis judex) to express a preference for the pastoral attributions of the diocesan bishop in terms of judgment of nullity of canonical marriage, in competition with those of the interdiocesan ecclesiastical tribunals created for the implementation of the concordat regime in Italy (MP Qua cura), could propose in this matter, after the Gospodinoff case (Constitutional Court, dec. N. 1/77) and the Pellegrini case (European Court of Human Rights, sentence 20 July 2001), the risk of a further vulnus to the principles of due process, on the other hand recently renforced in the Italian constitutional order. In dwelling on this inconvenience, the Author observes that the Catholic Church expresses with this, once again, its serious cultural and political delay in relation to the modern systems with which interacts ; and wonders why the Holy See has not decided by now to abandon the institutes and doctrines of ecclesiastical public law with which, starting from the 16th-17th centuries, with intransigence worthy of the better cause is has too often continued to feed the claim to keep on regulate relations with the political authorities on the models, now obsolete and perishable, of the Christian regime. The Author takes the opportunity to indicate how, conversely, the model of the interdiocesan tribunals, even if imperfect, can and should be generalized, in order to begin to solve the set of problems that the lack of modernization of the canonical system proposes, on the subject of the judicial system, in comparison with the level of refinement reached by legislative policy and the science of law (at the state and interstate level), in terms of protection of human rights, in the field of administrative and criminal procedural law.
\end{abstract}

SOMMARIO. 1. Preambolo: perseverare diabolicum? - 2. (segue) I tribunali regionali nella attuazione del regime concordatario, in Italia - 3 . Ancora in tema di competenza matrimoniale dell'ordinario diocesano. Intermezzo - 4. Corollari di principio in tema di giurisdizione penale e/o amministrativa - 5. Problematicità di una transizione contraddittoria, a ogni livello - 6. (segue) Dalla frammentazione feudale all'armonia di un moderno ordinamento giudiziario. 


\section{1 - Preambolo: perseverare diabolicum?}

Come la storia moderna ha dimostrato, esiste un limite, oltre il quale ogni monarchia assoluta rischia di andare in frantumi; e questo accade allorché non è più in grado (o più non vuole) dare in qualche misura conto - senza deliri di onnipotenza - della razionalità di scelte destinate a incidere profondamente nel corpo sociale. È a questo livello che i regimi di democrazia esprimono la loro rispondenza a esigenze effettive di elasticità governante, laddove il loro è un regime che, in maggiore o minor misura, dà spazio e voce all'opposizione politica.

A suo modo, l'apertura in atto di un generale processo sinodale con la dichiarata intenzione di inserire appropriatamente il populus Dei nei processi decisionali sembra oggi andare nel senso di un allargamento della base del consenso ecclesiale; eppure, la sopravvivenza di certi modi di procedere per via di misure dal piglio borbonico - figlie forse della "età dei Pii" - rischia talora di contraddire la logica di quella tendenza egualitaria, che sola appare la più congeniale a quella che Rosmini, felicemente, definiva la "società teocratica" (dove, cioè, la sovranità appartiene a Dio solo).

L'intervento di Paolo Moneta $^{1}$ sul martellante attacco ai tribunali ecclesiastici interdiocesani in materia matrimoniale esprime, con l'intero peso che, nella Chiesa italiana, il parere di un tecnico fine ed esperto, e a un tempo prudente e moderato quale Egli è può garantire, tutta l'amara insoddisfazione della base laicale che conta - quella cioè che in tali tribunali ha speso se anziana, e/o sta investendo se giovane un tratto di vita professionale consistente - nei confronti di un indirizzo riformatore che, a causa delle sue innegabili lacune tecniche, appare francamente mal consigliato da qualcuno; se poi, addirittura, non ci si debba spingere a sospettare che i consigli di costui non finiscano per essere troppo sovente mal compresi, o non piuttosto vengano per caso (nella ipotesi che ci si ostini a non rendere più chiari e netti i profondi motivi dottrinali di una innovazione che incontra rilevanti opposizioni di merito) sistematicamente travisati da ignoti dilettanti ${ }^{2}$, indotti loro malgrado a stare al gioco di una debolezza del governo papale che nasce, al fondo, da una propria insufficiente trasparenza teleologica ${ }^{3}$.

${ }^{1}$ P. MONETA, Non c'è pace per i tribunali ecclesiastici regionali italiani, su questa Rivista, n. 1 del 2021.

${ }^{2}$ Del che sono forse indizio certe tonalità improprie del proemio dell'ultimo (si spera!) Motu proprio sul tema.

3 Oltre tutto, persistendo la Santa Sede nel comportamento attuale, il governo italiano 
A fronte di che, va ribadito che dei nuovi orientamenti, quali che essi siano, va reso in anticipo puntuale conto alla pubblica opinione, se si vuole che l'apparato gerarchico destinato ad attuarli (in questo caso, la magistratura specializzata costituita quasi un secolo fa sotto Pio XI, e riformata dal successore tramite il decentramento - di decisivo rilievo delle sue attribuzioni funzionali a tribunali regionali istituiti, in Italia, di concerto con l'episcopato) agevoli senza comprensibili resistenze la transizione verso un nuovo sistema sensibilmente diverso; del quale però, in atto, bisogna pur dire che il profilo strategico appare ancora incerto e/o, comunque, tutt'altro che coerente e chiaro ${ }^{4}$.

\section{2 - (segue) Sui tribunali regionali nell'attuazione del regime concordatario, in Italia}

Non è un mistero per nessuno che la trasformazione politica seguita nel sistema ecclesiastico italiano all'ineluttabile caduta (per debellatio) del potere temporale dei papi abbia visto l'inizio di una dolorosa crisi di orientamento per l'intera Chiesa universale: una crisi ideologica,

potrebbe anche essere costretto a chiedere, in via diplomatica, adeguate garanzie quanto al mantenimento degli impegni assunti nell'accordo di revisione concordataria del febbraio del 1984, in merito alla tutela del diritto di difesa dei cittadini italiani coinvolti in un giudizio di nullità di matrimonio; e ciò ai sensi dell'art. 8 , secondo comma, lett. b, dell'Accordo stesso, con il quale le clausole in merito dell'art. 34 del concordato del ' 29 vennero novate con pochi ritocchi, relativi proprio alla questione, dibattuta avanti alla Corte costituzionale, del rispetto delle formalità di un giusto processo in occasioni del genere.

4 Per una prima reazione fortemente avversa a misure ritenute frutto di una critica del sistema dato esagerata, o addirittura tendenziosa, si veda G. BONI, La recente riforma del processo di nullità di matrimonio. Problemi, criticità, dubbi (parte prima), in questa Rivista, $\mathrm{n}$. 9 del 2016. Contributo apparentemente redatto a caldo, ma integrato poi da interventi ulteriori della stessa mano, nell'insieme di accurato e ragguardevole peso argomentativo (nella stessa Rivista cfr. nn. 2 del 2017, n. 1 del 2018, n. 2 del 2018); seppure -a quel che risulta- finora del tutto inascoltati. Forse per il loro radicale porsi in contraddizione sistematica con l'azione legislativa del pontificato? Se ne trovano segni pure nell'ultimo numero della Rivista, a cominciare dall'intervento Ancora sul legislatore paziente o impaziente, in cui senza ambagi si ammonisce il Papa, implicitamente, a lasciare ogni iniziativa in merito ai circoli (reazionari), in cui è iscritta la crema della opposizione di Curia al governo papale: in particolare, alla c.d. Commissione Interprete, che d'altronde già minaccia interventi a gamba tesa in caso di crisi che si aprano a seguito di (tutt'altro che deprecate!) emergenze nelle condizioni di salute di un Papa forse un po' scomodo per chi -nello stile del duello a distanza del card. Gasparri con Pio X- non tollera contraddizioni da parte di chicchessia. 
istituzionale e infine giuridica. E che il lungo pontificato di Leone XIII abbia avuto il pregio di provocare quel necessario processo di elaborazione del lutto atto a indurre a una metabolizzazione costruttiva dell'evento; che da noi ha comportato man mano - su un terreno di sano realismo - innovative proposte di traslazione su un terreno di internazionalità pattizia del tema cruciale dei rapporti che, in un contesto toto coelo diverso, Chiesa romana e comunità politica avevano intessuto all'interno della cristianità medievale e tridentina. Ma è, del pari, vero che, all'interno di tale sistema, era destinato necessariamente a sopravvivere qualche statuto autonomo per la figura del Papa come primate d'Italia, animatore della communio tra i vescovi italiani e fonte legittima di diretto impulso verso le manifestazioni collegiali di questa; al che va ascritta la tendenza a una tutela rafforzata che, anche in termini di tecnicismo giuridico, venne a supportare l'innovativo, delicato sistema ottenuto in Italia quando, tramite l'art. 34 del Concordato del 1929, il sacramento del matrimonio veniva a riottenere efficacia civile e, con esso, le controversie sulla sua validità tornavano all'esclusiva competenza del giudice ecclesiastico.

Si deve, quindi, pensare che l'osservanza di una regola non scritta di rispetto della cultura positivista all'epoca dominante avesse reso necessario - anche sul versante canonistico - un primo adeguamento formale alle esigenze dell'ordine pubblico statale in tema di controversie destinate, almeno all'epoca, a incidere sullo stato personale di larga parte dei cittadini; adeguamento pacifico per decenni in grazia anche a riforme di pregio tecnico indiscutibile, che oggi vengono messe in discussione senza che una previa dialettica interna abbia messo in luce - de iure condendo - i motivi di logoramento della graduale caduta di rilevanza delle riforme stesse: in seguito al tratto di tempo poi trascorso, col parallelo, prevedibile maturarsi di esigenze più ampie e imperiose di laicità, da parte della giurisprudenza costituzionale, italiana e comunitaria. Una caduta di stile poco perdonabile a un papato solitamente alieno da atti di autoritarismo, specie nei confronti di ambienti certamente leali, eppure adusi a serbare in ciò il senso critico proprio delle aristocrazie intellettuali e tecniche; senza le quali rimarrebbe certo fermo il valore dei vettori storici profeticamente intuiti da questo Papa in termini di soteriologia del Regno, ma è pure vano cullarsi, al di fuori di un loro convinto consenso intelligente, nell'illusione di poter conseguire, per mero intuito di innovazione dottrinale, obiettivi di innovazione duraturi in termini di razionalità e di durata. Criticare dall'esterno - ma senza assumersi responsabilità, neppure in termini di legislazione transitoria, di fronte ai problemi di continuità posti in concreto da una prassi quasi centenaria - 
può finire facilmente per rasentare l'apparenza di una condotta incompetente; così da correre il rischio di rinverdire, nell'utenza, il severo ammonimento di Alberico Gentili: Silete theologi, in messem alienam! 5

\section{3 - Ancora in tema di competenza matrimoniale dell'ordinario diocesano. Intermezzo}

\footnotetext{
${ }^{5}$ Altro e incomparabile discorso sarebbe stato quello fondato su una revisione più consistente dei postulati classici di un equilibrio oramai diverso con la ricerca sulle convinzioni della comunità delle origini (risultato critico di una nuova esegesi postmedievale delle fonti, in relazione con rilevanti mutamenti di prospettiva contemporanei sulla sessualità umana, diversi e distanti dal platonismo agostiniano): alla ricerca cioè di quella attenzione pastorale profonda ai bisogni delle persone in stato di coniugalità problematica, specularmente opposta all'ossessiva ricerca di un vizio (di forma, o del consenso, o da impedimento) situato necessariamente nel presunto atto-fonte sacramentale di un rapporto - quello di coniugio - frutto viceversa processuale di una vicenda affettiva singolare e irripetibile, in qualche modo ben delineata nelle fonti di Graziano; e in tale storia variamente situato in virtù sia delle idee sul matrimonium initiatum (nelle varie versioni della copulatheorie), sia di quelle richiamate da una visione della violenza, che tenga in maggiore conto anche la pressione del contesto socioculturale, in cui il vincolo coniugale sia venuto man mano a costituirsi. Con ciò, entrando non solo in dialogo con la oikonòmia ortodossa, ma perfino con l'atteggiamento dell'Apostolo delle genti, dedotto dalla fonte autentica di un suo decreto di nota e indiscutibile indulgenza dispensativa, ben oltre il favor fidei del gratuito rigorismo della sua recezione medievale; da cui la Roma della Rinascenza gesuitica trasse in seguito ragione per derivare, in virtù di analogia, le molteplici figure di dispensa "petrina" introdotte a partire dal secolo XVI nell'orizzonte di una rivalutazione serena e pacificata della felicità coniugale [in tal senso, vedi già le proposte abbozzate nelle mie Ipotesi di sviluppo della dottrina del matrimonio dopo il concilio Vaticano II, comunicazione in La Chiesa dopo il Concilio, Atti del Congresso internazionale di Diritto canonico (Roma, 14-19 gennaio 1970), Giuffrè, Milano, 1972, vol. II/2, p. 1427 ss.; intervento invano richiamato con sincero sostegno, qualche decennio dopo, dal compianto amico e collega Luciano Musselli]. Riserve queste di saggezza pastorale, disdegnate forse da chi predilige più impervie e saccenti vie speculative, percorrendo le quali "per apparer ciascun s'ingegna e face / sue invenzioni; e quelle son trascorse / da' predicanti e 'l Vangelio si tace". Là, dove Dante conclude la propria perorazione indignata ("non ha Fiorenza tanti Lapi e Bindi / quante siffatte favole per anno / in pergamo si gridan quinci e quindi") non senza avere spietatamente demolito l'albagia degli scolastici con altre due taglienti, corrosive terzine: "Sì che laggiù, non dormendo, si sogna / credendo e non credendo dicer vero; / ma né l'uno è più colpa e più vergogna. / Voi non andate giù per un sentiero / filosofando; tanto vi trasporta / l'amor de l'apparenza e '1 suo pensiero! / E ancor questo qua sù si comporta / con men disdegno, che quando è posposta / la divina scrittura, o quando è tòrta" (Divina Commedia, Paradiso, canto XXIX, 93 ss.).
} 
Il nuovo contesto legittimava bensì, quindi, un intervento di franca riduzione di pregiudizi dottrinali consolidati in una fase esegetica ancora immatura; ma non perciò esso avrebbe dovuto muovere - a parere di chi scrive - per la indiretta linea, organizzativa, della riforma procedurale: correndo così il rischio, certo gratuito, del sospetto di un atto di astuzia "modernista". Ché poi, a maggior ragione, un tale sospetto non avrebbe mancato di rafforzarsi passando per vere e proprie castronerie storiche, come quella ${ }^{6}$ secondo cui il vescovo sarebbe l'esclusivo giudice naturale di questo tipo di controversie. Laddove esso potrebbe, viceversa, diventarlo in Italia all'interno di una trasformazione equitativa della giurisdizione matrimoniale pensata da Benedetto XIV, che passi per un canale gemello, parallelo a quello attualmente egemone ma che da esso sia distinto nettamente: istituendo un vero e proprio percorso organizzato e gratuito di giurisdizione volontaria 7 in materia, magari connesso con spazi per una trattazione alternativa della crisi coniugale, in un contesto di accoglienza fraterna di essa in ambienti di decantazione (anche tecnico-terapeutica) del "tentativo di conciliazione"; ambienti a prevalente composizione laicale, tali da fugare i dubbi sull'idoneità alla funzione, proponibili forse nei confronti di soggetti pastorali celibi.

In un orizzonte del genere - cioè in termini di diaconia prudenzialmente articolata in tutte le sue diramazioni da un legislatore avveduto - la Chiesa potrebbe perfino ritrovare un suo ruolo autentico di ministra di ascolto e di pace nelle crisi matrimoniali; il che è probabilmente nei desideri profondi dell'attuale vertice del governo ecclesiastico. Ma non è chi non veda come un'impresa del genere, se iscritta nella logica concordataria dell'apprestamento di verdetti canonici preordinati alla assunzione di efficacia di giudicato "esterno" dei medesimi, mobiliti risorse culturali e tecniche la cui complessità postula il ricorso a istanze di taglio inevitabilmente collegiale e sopradiocesano (con

6 Esemplare, sul punto, G. RABINO, Ipse episcopus iudex: ritorno alla tradizione canonica?, su questa Rivista, n. 7 del 2017.

7 Del resto, non si dà già da oggi, all'interno dei cann.1686 ss., la possibilità di trattare una questione di vera e propria nullità di matrimonio in una sede tipica di giurisdizione volontaria? E, nel tempo, non si è configurata immediatamente come giurisdizione volontaria l'intera materia dello scioglimento del matrimonio degli infedeli per privilegium fidei? E, in tal materia, non si è data una progressiva commistione (quando non un rovesciamento) con le esigenze dell'amore coniugale in rapporto alle formalità di interpellazione, surrogate frequentemente da informative ex fama (dummodo constet, etiam summarie et exrtraiudicialiter, coniugem absentem moneri legitime non posse etc.)? 
conseguente sacrificio dei risvolti pastorali delle questioni a esigenze tuzioristiche ${ }^{8}$ proprie degli ordinamenti esterni di riferimento).

\section{4 - Corollari di principio in tema di giurisdizione penale e amministrativa}

Quanto alla giurisdizione criminale, già altrove mi è capitato di indicare, su questa Rivista ${ }^{9}$, il punto opportuno di caduta di un razionale riparto della materia tra ministero episcopale di riconciliazione e via contenziosa di cognizione ${ }^{10}$ repressiva dell'illecito penale in nolentes. E nulla e nessuno potrà convincermi a recedere dalle opinioni espresse, in quel contesto, a favore d'una più netta possibile separazione, in primo luogo valoriale - e solo conseguentemente di competenza - nell'autonomo, e differenziato approccio di merito, che l'illecito colpevolmente consumato dal reo propone, nel contesto ecclesiale, all'intervento autoritativo della comunità, del resto in linea di continuità con una tradizione rabbinica inveterata ${ }^{11}$.

\footnotetext{
8 Il che non è avvenuto a proposito dell'affrettato deferimento (dopo la De delictis gravioribus) al Dicastero dottrinale della repressione dei fatti di pedofilia; della quale solo ora stanno emergendo i risvolti di incivile mattanza, operata anche tramite reclutamento di personale completamente digiuno di formazione giuridica (e comunque senz'altro incapace di trattare questioni tanto delicate nel rispetto dei principi del giusto processo), in vista nel perseguimento sacrosanto -quando però non pressoché esclusivo, come invece sembra risultare- di obiettivi politici e di immagine istituzionale compromessa da riparare (per non parlare di episodi -tutt'altro che rari- di personale vendetta incrociata fra carrieristi!).
}

${ }^{9} \mathrm{Cfr}$. Tra conversione del peccatore e punizione del reo. Le contraddizioni del processo penale canonico, oggi, in questa Rivista, n. 12 del 2020, p. 126 ss.; cfr. pure L'Inquisizione come tipo di giudice immune da intuitus personae. Una alternativa per il superamento dell'attuale crisi dell'udienza penale diocesana e per quella della giustizia amministrativa, n. 1 del 2021, p. 216 ss.

10 Oltre tutto, il controsenso dell'identità di livello tra la fase della riprensione esortativa del (presunto) reo e quello della successiva formalizzazione inevitabile di una ipotesi di reato da punire nei suoi confronti comporta rischi sul piano di comportamenti ipocriti del reo nella prima fase e, nell'altra, di vissuti possibili di risentimento vendicativo da parte del vescovo, che nella prima fase si sia appena sentito respingere i paternalistici tentativi di composizione esperiti nei confronti dell'indiziato non confesso: situazione questa aberrante, tale da risultare comunque enutritiva peccati.

11 Il tema venne trattato a fondo, nella cornice delle giornate teramane del 2004 (Forme stragiudiziali o straordinarie di risoluzione delle controversie nel diritto comune e nel diritto canonico), in una perspicua relazione di G. BARBAGLIO, Dic ecclesiae... (Math. 18,15-17). Spunti storico-critici. Ne emerse tutta la specificità dell'approccio ecclesiale alla crisi rappresentata dalla flagranza di una colpa contro il prossimo; con differenziazioni interessanti tra una piattaforma di pura esegesi biblica e gli sviluppi dell'istituto 
Non troppo diverso discorso, per altro, mi sembra porsi a proposito dell'esercizio arbitrario del potere amministrativo; che pure ha conosciuto approfondimenti di rilievo - rispetto a importanti precedenti, formulati in termini durante i pontificati di Pio X e di Paolo VI - alla vigilia della ben nota redazione del codice del 1983, che conteneva un titolo sui tribunali amministrativi; titolo che il tratto di penna di un ben noto ukase del papa polacco cassava, riportando perentoriamente al 1917 le lancette dell'orologio della giustizia amministrativa nella Chiesa ${ }^{12}$. Tema di riforma, evidentemente, che ha mostrato di avere avversari ben più potenti ${ }^{13}$ di quelli che alla revisione del sistema penale si oppongono, ma più che altro per piatti motivi di continuità con la prassi tridentina $\mathrm{e}$ postridentina.

Altrove $^{14}$ ho ricordato con insistenza come l'età moderna abbia assistito a una trasformazione radicale delle modalità di rapporto tra individuo e istituzione nella storia del cattolicesimo romano. E, forse per questo, la Commissione pontificia di revisione del CIC del 1917 aveva ritenuto opportuno frapporre una sorta di interlocuzione bonaria sopravvissuta alla cancellazione dell'istituzione dei tribunali amministrativi regionali - tra l'emissione di un provvedimento e la sua

constatabili nella storia successiva (esemplare come parametro a contrario P. BELLINI, L'obbligazione da promessa con oggetto temporale nel diritto canonico classico, Giuffrè, Milano, 1964).

${ }^{12}$ L'evento è noto, così come la sua universale riprovazione da parte della canonistica mondiale, fatta eccezione per gli gnomi del potere assoluto, annidati per lo più nella Commissione "Interprete" e nei vertici della Segnatura Apostolica. Due centri di potere, che il papa regnante farebbe bene a ridimensionare $(\mathrm{e} / \mathrm{o}$, ancor meglio, a eliminare dal novero degli organi costituzionali del sistema).

${ }^{13}$ La blindatura del "sistema Gasparri" di insindacabilità giudiziaria dell'Esecutivo nei suoi più vari comparti, collocati al centro e/o alla periferia dell'ordinamento amministrativo, è a un tempo dottrinale, ideologica e politico-costituzionale. Praticamente uno di quei dogmi indiscutibili, al riparo dei quali ogni sopruso ama presentarsi nella storia.

${ }^{14}$ Cfr. qui Tra città di Dio e società "perfette". Il linguaggio del diritto pubblico ecclesiastico come cifra ricostruttiva della transizione cattolica dal mito alla modernità, su questa Rivista, n. 7 del 2017. E ancora ivi (n. 9 del 2017) Da governo secondo diritto a regime determinato dalla forza. Sulle ascendenze della autocrazia papale tra i 'Dictatus Papae' e la Controriforma; e sulla parabola finale della tirannide 'benevola' del Bellarmino; con tesi poi ribadite in Dalla tirannide "benevola" del Bellarmino all'autocrazia burocratica del Gasparri (ivi, n. 42 del 2017), passim. Fino alle conclusioni perentorie, forse esasperate, de I guardiani del 'golpe' di Gasparri, dal card. Pericle Felici in poi. Parabola dei codici del Novecento: il 'sistema romano' dal fascismo al fascismo (ancora sull'inesistenza di diritti dei fedeli nella Chiesa), ivi, n. 11 del 2018. 
(eventuale) conferma da parte dell'Autorità emanante ${ }^{15}$. Per contro, è la violenza stessa del potere espresso nell'atto provvedimentale a ribaltare da sola l'equilibrio tra le parti in causa: unilateralmente esponendone la più debole - destinataria di un atto imperativo - a un affievolimento anticipato della propria posizione giuridica, in virtù della presunzione di legittimità dell'atto impugnato; mentre l'uscirne resta affidato al gioco incerto del ritiro del comando, o della sua sospensione, in via gerarchica o per atto amministrativo di spontanea autotutela. Aporie, queste, da cui questa materia sarebbe stata risparmiata sia alla luce del progetto (di Pio X) di confermarne l'attribuzione giudiziale alla Rota, sia della coeva proposta del Decano della Rota (il card. Igino Lega, processualista insigne) di affidare l'intero contenzioso amministrativo a un Giudice speciale ${ }^{16}$;

15 Lo strumento collegiale di conciliazione di cui al can. 1733 non pare, in realtà, che venga utilizzato apprezzabilmente neppure laddove risulta stabilmente istituito, a livello di conferenza episcopale, o diocesano. D'altronde, la strettezza dei termini per la rimostranza e per l'impugnazione restringe considerevolmente gli spazi di intervento degli arbitri, o dei mediatori, volto a individuare una soluzione alternativa al dissidio aperto. D'altro canto, il sospetto di una collusione obbligata tra Amministrazione resistente e Autorità gerarchica riceve di solito conferma nella prassi; mentre il livello di Curia è decisamente troppo lontano da quello in cui la lite è sorta, per essere poi istruita e decisa (quando poi non maturino nelle more, col loro automatico determinismo, i tempi del silenzio-rigetto). Non risultando d'altronde messo in atto altro meccanismo di coordinamento (salvo quelli, tenui e indiretti, di cui al can. 1736) tra la via conciliativa e quella contenziosa, diventa inevitabile che quest'ultima prenda il sopravvento, prima che le parti trovino una seria linea di dialogo. Né all'organo di conciliazione è attribuita nei preliminari autorità (neppure monitoria), quale quella spettante al giudice in contentiosis ai sensi del can. 1446, mediante la facoltà di esercitare pressioni nel senso di una soluzione mediatoria, o transattiva (sarebbe ciò, forse, avvertito come lesa maestà da un Esecutivo onnipotente?).

Non sono da escludere però, de iure condendo, modifiche volte a rendere meno elusivo lo scenario di questa inedita versione del si peccaverit in te frater tuus; versione cui potrebbe dar luogo perfino l'ingiusta sopraffazione dell'innocente, o la sua diffamazione calunniosa, entrambe magari perpetrate dolosamente a mezzo delle solennità provvedimentali, senza ombra non solo di scrupolo, ma pure di rispetto sia del diritto di difesa del destinatario, sia di quello alla sua buona fama, magari fino a quel punto immacolata...

${ }^{16}$ Mezzo secolo dopo, la seconda era la via prescelta da Paolo VI, al fine di assicurare al colpito da un provvedimento illegale un giudice e un giudizio; ma la sede prescelta nella Segnatura non offriva garanzie sufficienti di imparzialità; mentre la limitazione del vizio deducibile alla violazione di legge, parificando il rimedio a una mera querela per un vizio dell'atto in procedendo, e/o in decernendo, svuotava la cognizione dello stesso di qualsiasi incidenza inquisitoria sul terreno (teleologico) dello sviamento di potere! Ma per maggiori dettagli su alcune decisive linee di fondo della politica legislativa di Paolo VI, su questa Rivista (n. 11 del 2020) cfr. il mio Sui principi di dogmatica costituzionale 
proposte tutte eluse, o accantonate a suo tempo dal Gasparri con abili temporeggiamenti, in attesa di portare a termine il colpo di mano costituzionale che andava maturando in pregiudizio della Rota, e al tempo stesso della separazione dei poteri del sistema ${ }^{17}$, nel sessennio seguito alla promulgazione del codice piano-benedettino ${ }^{18}$.

\section{5 - Problematicità di una transizione contraddittoria, a ogni livello}

Svincolarsi dal terreno del mito delle origini è necessario, se si intende davvero operare consapevolmente nella semplice, ma articolata realtà di quell'actus trium personarum, che è detta processo. Il che affermo necessario, per certa canonistica di Curia, anche per aver acquisito, ultimamente, prova documentale che, a palazzo del S. Uffizio, non è ancora nota - con buona pace del Concilio e di un memorabile, eppur pacato intervento di Giuseppe Capograssi su legge e caverna - la differenza tra processo e linciaggio ${ }^{19}$. La notizia inedita di tanto

compatibili con le dinamiche di potere interne alla vita della Chiesa e alla storia dei loro mutamenti. Idee e problemi del passato e del presente.

17 Anche qui è valido tuttora il richiamo ai già citati interventi Sulla "scomparsa dei diritti" nella Chiesa post-tridentina. Frammenti di una mutazione epocale nella pace delle istituzioni ecclesiali (su questa Rivista, n. 6 del 2020); oltre a Genesi e vicende dello Jus publicum ecclesiasticum nell'età postridentina. Una risposta romano-cattolica alla modernità (ivi, n. 4 del 2020). Alle cui note di sconforto si aggiunge ora il mio ultimo saggio per la rivista "Il tetto" di Napoli: Tra Aristidi e Polifemi. L'arduo appuntamento delle chiese con la propria riforma (in particolare, del cattolicesimo romano). Lo si veda anche su questa Rivista, n. 3 del 2021).

18 È stato poi da queste prime, autorevolissime indicazioni alternative alla "dottrina Gasparri" che doveva prendere le mosse, dopo il Vaticano II, la proposta di istituzione dei tribunali amministrativi interdiocesani, che si colloca pienamente nel quadro della VII direttiva del sinodo dei vescovi del 1967; mentre in Italia troverebbe una magistratura e una avvocatura pronte da utilizzare (previi per il personale adeguati percorsi formativi differenziati di addestramento e tirocinio in materia amministrativa e penale, da espletarsi preferibilmente sotto la supervisione e il controllo congiunto del Decano della Rota, del Rettore dell'Ateneo Lateranense e del Presidente del Tribunale dello S.C.V.) proprio in quei tribunali interdiocesani testé screditati come un residuo arcaico dal quale liberarsi, o per lo meno da ridimensionare.

19 Sul punto, riservatezza professionale vuole che, per ora, la prova documentale in discorso non sia propalata; eppure la sua stessa esistenza mostrerebbe che i processi de delictis gravioribus, deferiti a suo tempo al Dicastero dottrinale in unico grado, potrebbero essere stati sovente celebrati senza previa contestazione degli addebiti al prevenuto, né vocatio in ius del medesimo, e a fortiori senza menzione del suo diritto a munirsi di un difensore di fiducia in apertura di istruttoria. Con la conseguenza che, dell'intero 
sbalorditiva arretratezza fa il paio con quella che, almeno poco oltre metà degli anni 2000, verdetti del genere neanche erano suscettibili di impugnazione alcuna! Sembra dunque indifferibile, a questo punto, cominciare a praticare le vie di un radicale svecchiamento della "cultura" giuridica che abita i recessi, da cui aberrazioni del genere provengono ${ }^{20}$; quanto meno obbligando chi assume di "rendere giustizia" al loro interno a rassegnarsi a farlo su un terreno di liceità rispettando, con assoluto rigore, i principi del giusto processo ${ }^{21}$.

Tanto premesso, merita considerazione toto coelo diversa, sul piano del buon andamento di questo delicato settore, la tradizione con cui il sistema legislativo ha preso da quasi un secolo ad aggregare in sedi collegiali interdiocesane moduli organizzati di giurisdizione ordinaria come quelli che, pur ampiamente collaudati, sembrano, alla collega Boni e non solo, presi di mira - con vigore degno di miglior causa - da linee riformatrici messe in atto dal papato attuale secondo modelli istituzionali la cui temperie pastorale innovativa è incontestabile; ma che non possono senza imprudenza pretendere di spedire in soffitta, archiviandoli, gli organi giudiziari precedenti, sol che si voglia procedere, con consapevolezza storica, a quello sforzo di armonizzazione degli opposti che, nella ricostruzione sistematica degli istituti, è il compito talora arduo (cui opporre un non liquet è comunque vietato all'interprete) assegnato alla dottrina e da realizzare, con disciplina, sullo sfondo - bettiano - della totalità e coerenza della considerazione ermeneutica ${ }^{22}$.

processo e della sua conclusione (radicalmente incidente sul suo stato personale), l'interessato abbia soltanto finito per apprendere quanto succintamente comunicatogli $a$ posteriori, nel dispositivo del decreto di condanna nel merito.

${ }^{20} \mathrm{Mi}$ rendo conto benissimo che qualche Eminenza "mi starà in cagnesco", tanto per cambiare, per i "formalismi" cui richiamo al dovere di non rinunciare; ma non è colpa mia se - dietro certi inammissibili abusi - avverto piena la verità del commento di un processualista amico (all'epoca segretario nazionale dell'U.G.C.I.!), che ravvisava in una mentalità diffusa nella Chiesa, non solamente gerarchica, il segno di una forte propensione verso la retorica accusatoria, per contro troppo facilmente ignara delle cautele imposte dalla prudenza istruttoria ....

${ }^{21}$ Ancora una volta ha trovato conferma, in tale occasione e a proposito del diritto di difesa dell'inquisito, un duplice tradimento storico, da parte del Dicastero qui indicato, nei confronti sia delle direttive collegiali di principio impartite a suo tempo dall'ultimo concilio e dal sinodo dei vescovi del 1967, sia perfino della stessa Integrae servandae di papa Paolo VI. Si tratta a mio avviso di una deriva inquietante, alla quale non ritengo che possa essere ritenuta estranea la posizione apicale per troppo tempo esercitatavi da un intellettuale non giurista, come il card. Ratzinger. A eccessi del genere mai si sarebbe pervenuti, quando il S. Uffizio era retto da canonisti del calibro di Alfredo Ottaviani!

22 Oltre tutto, in tutta la polemica accesa da una retta coscienza professionale 


\section{6 - (segue) Dalla frammentazione feudale all'armonia di un moderno ordinamento giudiziario}

A parere di chi scrive è da credere, d'altronde - checché si opini quanto al "dogma" della inderogabilità della competenza di primo grado del vescovo - che le vedute tradizionali in voga quanto a un riparto razionale delle attribuzioni degli organi giudiziari dipendano assai più dal supino ossequio a convinzioni mitiche derivate da una concezione discendente del potere ecclesiastico (ripartito, dalla riforma gregoriana in poi, secondo la duplice linea del sovrano feudale e dei vescovi-conti), storicamente situata e manifestamente incompatibile con i principi di collegialità accolti dall'ultimo concilio. Ma che manchi tuttora uno sforzo comune tra i canonisti, volto a trarre da ciò tutte le conseguenze, che una simile rivoluzione culturale comporta sul terreno istituzionale. Intendo, in primo luogo, riferirmi alla urgente necessità di convincersi a considerare la funzione giudiziaria all'interno dell'ordinamento generale come una "variabile indipendente" dalla condizione dei rapporti tra gli organi costituzionali; il che si evince dal suo riferirsi, oltre tutto, a una dimensione non di potere storicamente situato, ma di "ragione senza passione", bisognosa solo del rispetto dovuto alla sua indipendenza e imparzialità.

Se si accetta un postulato teorico-politico di tal genere, allora non vi è più luogo per mal certi agganci a questo, o a quel potere costituzionale. La giurisdizione è, e tanto basta per capire come organizzarla, senza lasciarsi agganciare da obiezioni improprie, legate alla ricerca di nuovi equilibri, appropriati alle dinamiche della lotta politica in corso da sempre tra papa, vescovi e sedi collegiali di coordinamento tra il centro e la periferia del sistema.

Il che significa che le circoscrizioni giudiziarie dovranno essere quelle che, di volta in volta, appaiano le più congeniali e conformi alle esigenze dell'utenza: proprio come esemplarmente avvenuto, del resto, in

ingiustamente offesa, è per altro il caso di rilevare che i colleghi intervenuti hanno mancato di situare le mosse di politica legislativa in corso nel contesto di una sua ponderazione all'interno di una logica di "diritto mite" resa necessaria, secondo un apprezzamento insindacabile del legislatore, da una lettura dei segni dei tempi appropriata a una fase decisamente transitiva e problematica dei rapporti tra chiesa e mondo; tale da restituire, pur tardivamente rispetto al merito scientifico dell'Autore, rilievo decisivo a una svolta esegetica come quella, volta a una rinnovata ricerca pastorale di senso sul mistero della coniugalità, che ha impegnato l'intera vita di ricerca del mio amico G. CERETI, fin da Divorzio, nuove nozze e penitenza nella chiesa primitiva, Dehoniane. Bologna, 1977 (ora per le edizioni Aracne, Roma, 2013). 
occasione di quel completamento ideologico, politico e tecnico dell'art. 34 del Concordato, che la Qua cura di Pio XI è stata efficacemente, ai suoi tempi.

A partire da presupposti di tal genere, sarà una questione di merito decidere a quale comparto costituzionale (se non altro per questioni di bilancio) attribuire questa o quella funzione giurisdizionale; e sarà una questione di merito decidere se mantenere condizioni di compatibilità (a livello diocesano, o collegiale), tra il nucleo delle funzioni legate al contenzioso matrimoniale attuale e quello - a trattazione "mite" comunque investito da attività miste, più appropriate a misure di giurisdizione volontaria.

Quanto al resto degli orizzonti possibili in un contesto di autoritarismo in globale declino, si esprime qui l'opinione che la sede diocesana sia - in linea generale - senz'altro la più appropriata alla trattazione di momenti di delibazione non contenziosa degli affari in lite; ma che la sede dell'area regionale ecclesiastica sia senz'altro da preferirsi a fronte di questioni disciplinate da regole vincolanti di diritto, soggette a interpretazione da affidarsi a operatori consapevoli - anche a livello di relazioni interordinamentali - delle più aggiornate condizioni esistenti di sussidio dottrinale e giurisprudenziale.

Ciò con tutte le ovvie generalizzazioni razionalmente deducibili, in materia sia di contenzioso penale, che di contenzioso amministrativo; ma previa definitiva sottrazione, comunque, alla decisione esclusiva dell'Autorità dell'opzione tra coercizione disciplinare e giudiziaria: così da escludere la possibilità che il diritto di difesa del fedele sia comunque esposto al rischio di vessazioni arbitrarie, anche in sede gerarchica, da parte di qualche sprovveduto (e magari un po' isterico) "sceriffo" dell'Esecutivo.

Il tutto in uno scenario finalmente conveniente a una temperie storica che ha deciso definitivamente, da gran tempo, di farla finita coi fascismi del secolo breve; e che chiede - salva l'insindacabilità degli atti politici - che, in via di massima, l'ultima valutazione vincolante dei rapporti controversi sia restituita, anziché al recondito intento di un potente, alla pubblica e manifesta serenità di un giudice (la Rota, o una magistratura speciale); l'adempimento della cui sentenza venga sentito come rispondente al supremo interesse pubblico della salvaguardia dell'ordine costituito. Inteso, quest'ultimo, come complessità governante (a un tempo dunque istituzione e ordinamento, per dirla con Santi 


\author{
Romano), comprensiva quindi della considerazione - ovviamente \\ impreteribile - dell'equità canonica non scritta ${ }^{23}$.
}

${ }^{23}$ Cfr. can. 1752 del CIC del 1983. Infine, a conclusione di un intervento del quale -a differenza di quelli, senz'altro rispettabili, dei colleghi Boni e Moneta- mai potrebbe dubitarsi dei convinti presupposti anticoncordatari di partenza (radicati come sono nella tradizione della Sinistra cristiana), proprio motivi di equità canonica inducono qui a spendere qualche parola sull'odiosa insinuazione, con la quale qualche portavoce male informato ha avanzato il sospetto che i professionisti abilitati al patrocinio avanti ai tribunali interdiocesani si preoccupino per loro presunti 'privilegi' (oltre tutto risibili) e/o che i presidenti dei medesimi si oppongano alla Mitis iudex per interessi di potere. Questi interessi di certo esistono, ma trovano radice in un sistema (quello dell'onnipotenza cattolica) costituito per scelta politica della Santa Sede, or sono novant'anni, all'interno di una strategia culturale e politica globalmente volta a determinare un confine invarcabile tra i confini - militarmente difesi dai fascismi storici - di una cristianità (intesa come populus ductus da un ceto clericale dominante) e il resto del mondo. Di tale sistema le persone ora attaccate dall'ipocrisia della Curia romana, magari senza saperlo ci ricordano che ne fu frutto la saldatura tra art. 34 del concordato lateranense e art. 142 del codice civile del 1942: con la quale un regime liberticida spegneva ogni legittima speranza di veder ripreso nella tradizione italiana il principio -romanistico, prima che liberale sancito dall'art. 227 del Code Napoleon come rottura ideologica con l'Ancien régime, e pertanto laicamente irrinunciabile, della dissolubilità del matrimonio per giusta causa. Una rottura, contro la cui tardiva interruzione in Italia (tramite la legge Baslini-Fortuna), una improvvida CEI giocò la carta lacerante del referendum, per poi infierire su chi ravvisando nel quesito referendario un attentato alla libertà di coscienza dei non credenti - aveva preferito, fedele al vangelo e al concilio, obbedire piuttosto a Dio che agli uomini. Preoccupa, a questo punto, il persistere sistematico dell'intimidazione, come strumento di governo di un Esecutivo onnipresente e insindacabile: ma è un sistema che deve finire, archiviato tra i relitti della storia, seppure ancora vivo e vitale come mostra l'attuale vicenda. Ci si perdoni se, proprio nell'ansia di contenerne la virulenza, in queste pagine si è formulata la proposta di orientare il riformismo papale in direzione di un utilizzo non illiberale delle strutture giudiziarie istituite nella Qua cura di Pio XI: sperimentando in Italia un nuovo modo (prefigurato nel 1983, ma inattuato poi dal legislatore) di assicurare dignitosa tutela ai diritti della persona, nel processo penale e in quello amministrativo. Mediante istanze da proseguirsi in appello alla Rota il primo (fermo il principio della presunzione d'innocenza del reo fino al formarsi del giudicato); e differenziando la fase di gravame del processo amministrativo a seconda che si opti per il progetto di Pio $X$ (Rota), o per quello del card. Lega (giudice speciale, ma in posizione di indipendenza dall'Esecutivo). 